\title{
Effect of Chronic Low Back Pain on Balance and Functional Mobility in Women
}

\section{Khabeer Fatima1', Zahid Mehmood Bhatti², Iqra Khan³, Ubaid Ullah Akbar ${ }^{4}$}

${ }^{1}$ Physical Therapist, Ascenti: Physiotherapy Services, Portchester, Fareham PO16 8UZ, United Kingdom

${ }^{2}$ Associate Professor HOD, Bakhtawar Amin Medical and Dental College, Multan, Pakistan

${ }^{3}$ Assistant Professor/Consultant Physical Therapist, Bakhtawar Amin Medical and Dental College, Multan, Pakistan

${ }^{4}$ Assistant Professor, Lahore Medical and Dental College, Lahore, Pakistan

Author's Contribution

1-2Conception and design, Collection and assembly of data, ${ }^{2}$ Analysis and interpretation of the data, ${ }^{3} \mathrm{Critical}$ revision of the article for important intellectual content, Statistical expertise ${ }^{1-4}$ Final approval and guarantor of the article

Article Info.

Received: March 17, 2021

Acceptance: Dec 3, 2021

Conflict of Interest: None

Funding Sources: None

Disclosure: Article is retrieved from thesis

Address of Correspondence Iqra Khan

Email Id: Khan_iqra88@yahoo.com ORC Id: 0000-0003-1192-4842

Cite this article as: Fatima $\mathrm{K}$, Bhatti

ZM, Khan I, Akbar UU. Effect of Chronic Low Back Pain on Balance and Functional Mobility in Women. JRCRS. 2021; 9(2):71-74.

DOI: $10.53389 / J R C R S .2021090207$

\section{A B S T R A C T}

Objectives: To find the number of individuals with disturbed balance and impaired functional mobility, associated with chronic low back pain.

Methodology: It was a cross sectional observational study of seventy female patients with age 40 years or older being evaluated for disturbed balance and impaired functional mobility due to chronic low back pain. Data was collected from Bakhtawar Amin Memorial Trust Hospital from May 2019 to November 2019. The subjects were assessed through Berg Balance Scale for their disturbed balance and timed up and go test for their functional mobility. The data collected was statistically analyzed using SPSS 21 and displayed in forms of appropriate graphs in terms of percentages.

Results: Only one patient (1.4\%) was scored lowest $(0-20), 43$ patients $(61.4 \%)$ had moderate score (21-40) and 26 patients (37.1\%) had achieved maximum scores (41-56) on Berg Balance scale. The score for functional mobility on timed up and go test was normal (10 seconds or less) for 9 patients (12.9\%), 34 patients $(48.6 \%)$ had moderate impaired mobility and 27 patients $(38.6 \%)$ had greater impairment in mobility.

Conclusion: A high Prevalence of low back pain has been seen in the elderly women, which in turn effects the balance and functional mobility. These two being the major factors for independence, thus affecting the mobility. Age is markedly associated with the scoring on berg balance scale.

Key words: Balance, Chronic low back pain (CLBP), Functional mobility.

\section{Introduction}

Low back pain is a prevailing health problem worldwide that not only affects the individual's personal life but also impairs community roles immensely. It has been seen that females have the highest prevalence and also those individuals which are aged between 40 and 80 years. ${ }^{1}$ Low back pain is a common complain while dealing with the musculoskeletal issues, almost $4-33 \%$ of population is effected at some given point ${ }^{2}$. In case of chronic cases disability and activity limitation, Low back pain is more common cause second to arthritis ${ }^{3}$. Low back pain is related with disability and decreased functional mobility and it has been seen that its association with variation in balance is not generally emphasized.

Balance is mainly a biomechanical term which can be described as inability to uphold the line of gravity, that is a vertical line from body mass centre with minimum posture sway. ${ }^{4}$ Sway is unavoidable and necessary in some cases like body shifting from one foot to other foot, or while breathing and other minimal activities. Sway is also inevitable from external triggers like visual distortions or sudden floor transition. It is uncertain that increase in sway is a red light to the balance dysfunction. $^{5}$ To maintain adequate balance a number of 
systems like vestibular, somatic, sensory and visual, need to provide coordinated and organized input. Muscular imbalance and muscular weakness may also lead to disturbed balance. CLBP results in muscular weakness followed by pain. ${ }^{6}$

According to a study ${ }^{7}$ that was conducted to evaluate the relationship between back pain and balance, chronic backache has a direct linear link with an individual's balance. Chiropractors have already established a relationship between back pain and balance, whereas the linear connection has first time been established. The study recruited 210 patients with lower back, middle back and cervical pain.

Another study ${ }^{8}$ evaluated the differences in balance strategies in Chronic low back pain and healthy controlled subjects, using three dimensional motion analysis system.

A controlled study ${ }^{9}$ with follow up based on one footed and externally disturbed two footed postural control in patients with CLBP. The study compared normal healthy individuals at the beginning of functional back restoration programme. This study explains that impaired postural stability seems to be one factor in multidimensional symptomatic study of patients with CLBP. Postural stability is easily disturbed when there is impaired strength and coordination of muscles in lumbar and pelvic area.

Decrease in the neurological function related to age renders elderly people prone to falls and as a consequence sustaining injuries occur. Hence balance is critically important to prevent falls and injuries. A lot of work has been done on postural sway and studies are carried out. Balance is usually taken for granted when it comes in context with low back pain. So assessment of disturbed balance is the major consideration along with impaired functional mobility due to chronic low back pain to get improvement in treatment regimens of CLBP.

\section{Methodology}

It was cross-sectional observational study conducted on seventy female subjects aged 40 years or above. The subjects were evaluated on the basis of convenience sampling, for disturbed balance and impaired functional mobility due to chronic low back pain. The study setting was Bakhtawar Amin Memorial Trust Hospital, Multan and study duration was 6 months from May 2019 to November 2019. Sample size was calculated by WHO calculator in health studies. The parameters taken were confidence level being $90 \%$, anticipated population was $0.55^{10}$, the precision was 0.10 and the sample came out to be 67 .

The female subjects with forty years of age having chronic low back pain and those having history of more than six months were included in the study. Those with any history of surgical procedure of back in last six months, severe limitations in mobility, having terminal illness, neurological disorder and those having history of recent lumbar pain treatment were excluded.

Berg Balance Scale ${ }^{11}$ was then performed by the patient by doing 14 balance related tasks after demonstration from the investigator. It is used worldwide for the assessment of static or dynamic balance clinically. For functional balance tests, berg balance scale is the gold standard. It usually completes in 10 to 15 minutes and is consisted of various activities especially standing up straight from sitting position. Score ranges from zero (unable) to four (independent) depending upon the degree of achievement in each task. Total score is 56. Likewise Timed Up and Go Test ${ }^{12}$ was utilized to evaluate the functional mobility. This test checks the time a subject takes to get up from a chair, then walk normally for 3 meters, then turn back and walk to sit back in the chair. While performing the test, subjects are asked to be in their regular footwear and keep using their aid. Informed consent was taken and investigators educated the potential subjects/patients to ensure that they can reach a truly informed decision about whether or not to participate in the research. The data collected was statistically analyzed using SPSS 21 and displayed in forms of appropriate graphs in terms of percentages.

\section{Results}

Seventy samples were collected; 34 subjects were between 40 50 years, 26 patients had age between $50-60$ years, 8 patients were between $60-70$ years and 2 patients were above 70 years. Out of 70 patients $n=31,(44.3 \%)$ reported constant low back pain, $n=31,(44.3 \%)$ reported intermittent pain and $n=8,(11.4 \%)$ subjects were having dull pain. The duration of chronic low back pain was more than 6 months in $n=30,(42.9 \%)$ and $n=40$, (57.1\%) were suffering for 2 years.

The study described that out of $n=70$ patients, $n=18$, (25.7\%) had extreme difficulty while performing Activities of Daily Living, $n=46,(65.7 \%)$ subjects found it difficult with ADLs and $n=6,(8.6 \%)$ had little impact of Chronic Low Back Pain on Daily Living Activities.

From total of 70 patients $n=1,(1.4 \%)$ had lowest score $(0-20)$ on Berg Balance Scale scoring, $n=43,(61.4 \%)$ subjects had moderate score (21-40) while $n=26,(37.1 \%)$ have maximum scores (41-56) as shown in Figure 1.

Out of 70 subjects, $n=9,(12.9 \%)$ had normal score on Timed Up and Go Test scoring, $n=34$, (48.6\%) had disturbed 
mobility and $n=27,(38.6 \%)$ subjects needed assistance (Figure 2).

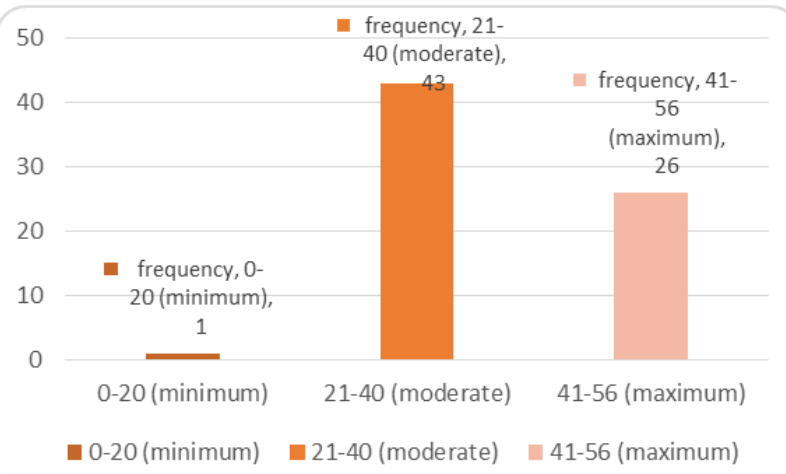

Figure 1. Frequency distribution for patient's score on berg balance scale

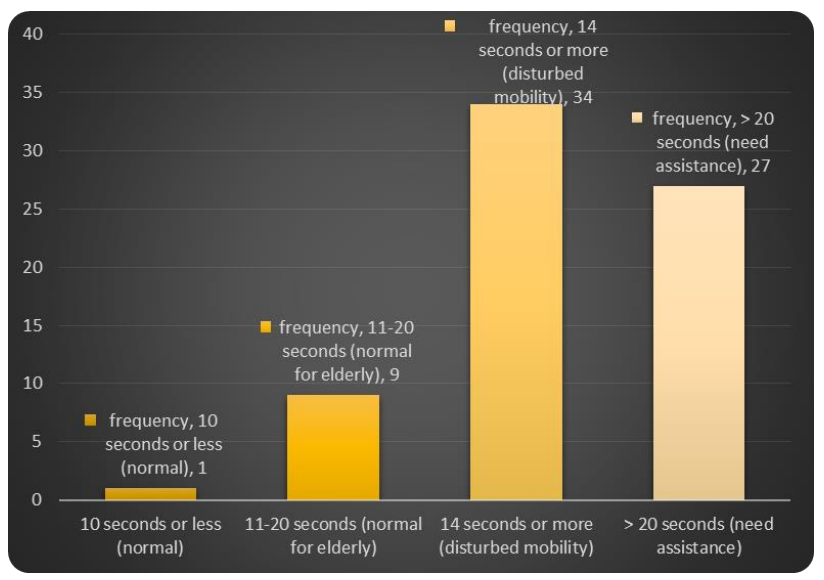

Figure 2. Frequency distribution for patient's score on timed up and go test.

\section{Discussion}

In the previous studies balance was evaluated specifically in those people having low backache in comparison with healthy individuals. The whole assessment and intervention procedures were carried out using force plates in upright standing position. The results showed increase in the mediolateral sway for low back pain subjects, specifically when task complexity was introduced during the assessment session ${ }^{13}$. Whereas the current study also targeted functional mobility along with balance in relation to low back pain.

In another study'4, two groups were compared (patients of CLBP and the control group), balance was measured in unstable sitting using three dimensional motion analysis systems and three dimensional angular displacements was estimated in 3 cardinal planes. A higher postural sway was observed in patients with CLBP compared to control group. The results of the mentioned study were in line with the current study.
Another study ${ }^{15}$ comprising of two groups reported one having CLBP and the other control group, postural sway was measured using force platform in one-footed and externally disturbed two footed stance. Impaired postural stability was observed just like the current study results.

One study was conducted to check the effects of CLBP on balance along with functional mobility in osteoporotic women above age of 65 years. Results of the study showed that balance and functional mobility were effected in the overaged females having back pain ${ }^{16}$. The results of the current study were correspondent with this previous study although no force platforms were used to assess the postural sway and disturbed balance. Instead a simple test was applied to assess the balance of the patients and functional mobility by another test.

\section{Conclusion}

In context with the obtained results it is noted that balance and functional mobility are markedly affected in chronic cases of low back pain particularly women above age of 40 and those having history of back pain more than 2 years.

Limitations and Recommendations: The sample size taken was small as compared to the general population of the city so the results which are obtained are more localized. To get the more generalized results this study must be carried out on a larger scale. Association should be done between variables like age and duration of pain with CLBP to get more information about influence of pain and balance. As it is concluded that balance and functional mobility are impaired in cases of low back pain, so special consideration must be given to balance and improvement of functional mobility in treatment regimen of chronic low back pain cases.

\section{References}

1. Foster NE, Anema JR, Cherkin D, Chou R, Cohen SP, Gross DP, et al. Prevention and treatment of low back pain: evidence, challenges, and promising directions. The Lancet. 2018;391(10137):2368-83.

2. Rajpurkar $P$, Irvin J, Bagul $A$, Ding D, Duan $T$, Mehta $H$, et al. Mura: Large dataset for abnormality detection in musculoskeletal radiographs. arXiv preprint arXiv:171206957. 2017.

3. Rishi $P$, Arora B. impact of muscle energy technique along with super-vised exercise program over muscle energy technique on quadratus lumborum and iliopsoas on pain and func-tional disability in chronic non specific low back pain. Int J Physiother Res. 2018;6(3):2748-53.

4. Lee B-S, Shim J-H, Choung S-D, Yoon J-W. Effects of Goal-Oriented Side Walking Training on Balance and Gait in Chronic Stroke Patients. PNF and Movement. 2018;16(1):93-103. 
5. Sadowska D, Krzepota J. Influence of posturographic protocol on postural stability sways during bipedal stance after ankle muscle fatigue. Perceptual and motor skills. 2016;123(1):232-43.

6. Zambare PD, Soni N, Sharma P. Effect of Cawthorne and Cooksey Exercise Program on Balance and Likelihood of fall in Older Women. Quadriceps Femoris Strength Training: effect of Neuromuscular Electrical Stimulation Vs Isometric Exercise in Osteoarthritis of Knee. 2015;9(3):55.

7. Ruhe A, Fejer R, Gänsslen A, Klein W. Assessing Postural Stability in the Concussed Athlete: What to Do, What to Expect, and When. Sports health. 2014;6:427-33.

8. Van Daele $U$, Hagman $F$, Truijen $S$, Vorlat $P$, Van Gheluwe B, Vaes P. Differences in balance strategies between nonspecific chronic low back pain patients and healthy control subjects during unstable sitting. Spine. 2009;34(11):1233-8.

9. Hodges PW, Moseley GL. Pain and motor control of the lumbopelvic region: effect and possible mechanisms. Journal of Electromyography and Kinesiology. 2003;13(4):361-70.

10. Wáng YXJ, Wáng J-Q, Káplár Z. Increased low back pain prevalence in females than in males after menopause age: evidences based on synthetic literature review. Quantitative imaging in medicine and surgery. 2016;6(2):199.
11. Downs S. The berg balance scale. Journal of physiotherapy. 2015;61(1):46.

12. Barry E, Galvin R, Keogh C, Horgan F, Fahey T. Is the Timed Up and Go test a useful predictor of risk of falls in community dwelling older adults: a systematic review and meta-analysis. BMC geriatrics. 2014;14(1):14.

13. Kent $P$, Laird $R$, Haines $T$. The effect of changing movement and posture using motion-sensor biofeedback, versus guidelines-based care, on the clinical outcomes of people with sub-acute or chronic low back pain-a multicentre, cluster-randomised, placebo-controlled, pilot trial. BMC musculoskeletal disorders. 2015;16(1):131.

14. Denteneer L, Van Daele U, De Hertogh W, Truijen S, Stassijns G. Identification of preliminary prognostic indicators for back rehabilitation in patients with nonspecific chronic low back pain: a retrospective cohort study. Spine. 2016;41(6):522-9.

15. Mok NW, Yeung EW, Cho JC, Hui SC, Liu KC, Pang CH. Core muscle activity during suspension exercises. Journal of science and medicine in sport. 2015;18(2):189-94.

16. Puszczalowska-Lizis E, Bujas $P$, Jandzis $S$, Omorczyk J, Zak M. Inter-gender differences of balance indicators in persons $60-90$ years of age. Clinical interventions in aging. 2018;13:903.

\section{Copyright Policy}

All Articles are made available under a Creative Commons "Attribution-NonCommercial 4.0 International" license. (https://creativecommons.org/licenses/by-nc/4.0/). Copyrights on any open access article published by Journal Riphah college of Rehabilitation Science (JRCRS) are retained by the author(s). Authors retain the rights of free downloading/unlimited e-print of full text and sharing/disseminating the article without any restriction, by any means; provided the article is correctly cited. JRCRS does not allow commercial use of the articles published. All articles published represent the view of the authors and do not reflect the official policy of IRCRS 